\title{
Automatic Fire Fighting Robo Vehicle Using Arduino
}

\author{
R.Brindha, S. Gomathi, K. Vanitha, R. E1anthirayan, A. Ananthichristy
}

\begin{abstract}
The firefighting is a dangerous field to work on . There are numerous conceivable outcomes a fire can begin in an industry or in any remote area. For example, in cotton factories, articles of clothing, fuel stockpiles, and so on. Electric spillages can prompt immense harm. Robotics is the emerging solution to protect human lives their wealth and surroundings. Hence we propose a fire fighting robot vehicle which can maneuver into locations humans cannot reach, automatically detect fires in its vicinity, alert people about the fire breakout and keep the fire within control before the actual firefighting rescue team is brought in.
\end{abstract}

\section{Keywords-Arduino, Robo vehicle}

\section{INTRODUCTION}

Our task as an Electrical engineer is to design and build a prototype system that can sense fire in small areas and alert the people of the existence of the fire for remedial actions We have also built in a water storage along with a pump to extinguish the detected fire to prevent it from spreading to other areas before the actual help arrives.

This mission has been divided into 3 stages and each task has been prototyped in the best way possible. Such as the autonomous movement of the robot involving obstacle avoidance, the automatic detection of fire in its periphery, the sounding of an alarm as it detects a fire and the control of fire spreading using water stored on the robot. Conventional Fire Fighting Equipment present

Fire fighting systems and equipment vary depending on the age, size, use and type of building construction. A building may contain some or all of the following features:

- $\quad$ Fire extinguisher.

- $\quad$ Fire hose reel.

- $\quad$ Fire hydrant system.

- $\quad$ Automatic sprinkler system.

\section{Limitations of the conventional methods}

A programmed fire concealment framework can control a fire in a working, as well as quench fires a long time before the fire division can touch base on scene. It does this without the requirement for any human mediation. But fire originated under a shelf area, which shields the heat from releasing upward toward the fusible links that actuate the automatic

Revised Version Manuscript Received on 10 September, 2019.

R.Brindha, Assistant Professor in SRM Institute of Science and Technology, Chennai, Tamilnadu, India.(Email: : brindha.apr16@gmail.com)

S.Gomathi,.K.Vanitha, Assistant Professor in St. Joseph's College of Engineering, Chennai, Tamil Nadu, India.

R.E1anthirayan, Assistant Professor in St. Joseph's College of Engineering, Chennai, Tamil Nadu, India.

A.Ananthichristy, Associate Professor in Saveetha engineering college, Chennai, Tamil Nadu, India. sprinkler extinguishment system, then the system would not detect the fire.

\section{PROPOSED FIRE FIGHTING SYSTEM FEATURES}

- The automatic fire fighting robot is a small scale version of what can be built for practical use.

- small size of the robot will allow it to go to inaccessible regions, where the fire is present

- To eliminate human intervention the robot automatically detects fire and extinguishes it to the maximum it can use its inbuilt water storage.

- It alerts the people about fires through alarm.

- Extinguishing agent was blocked from reaching the seat of the fire due to the location

- The robot keeps the fire in control and prevents it from spreading before major help is brought in.

\section{BLOCK DIAGRAM}

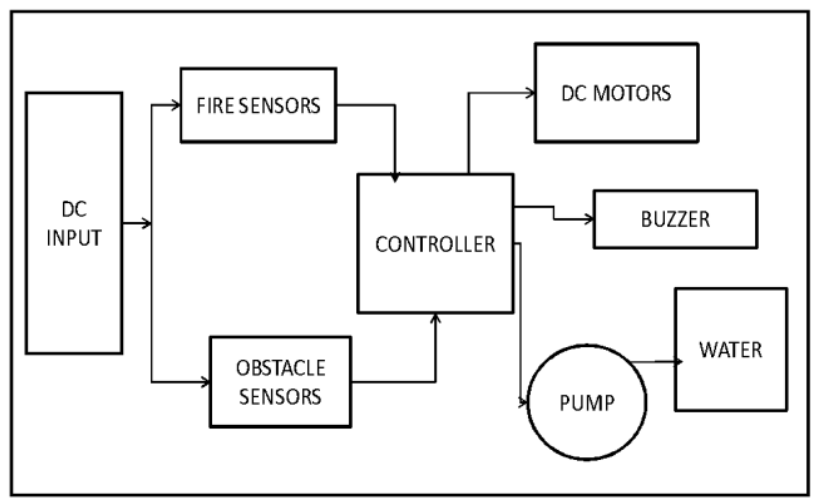

Fig. 1. Block diagram of automatic firefighting robo vehicle

\section{SYSTEM WORKING}

The circuit implemented consists of inputs which basically give data to the controller which is programmed to process the given raw information and do certain tasks depending upon the data

The input devices are powered using DC batteries so that the robot is free to move independent of any wired connections. Once the robot is turned on, the robot moves forward into the room, if there is any obstacle present then the obstacle sensor gives a high output to the controller; which then makes the wheels of the robot to turn in the opposite

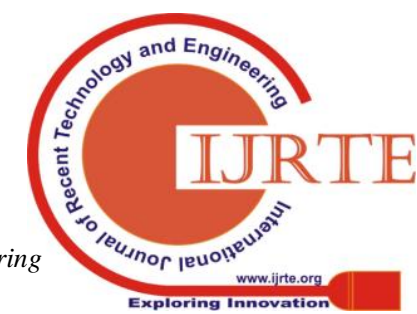




\section{AUTOMATIC FIRE FIGHTING ROBO VEHICLE USING ARDUINO}

direction and hence avoid dashing into the obstacle. While it is moving, the fire sensors keep monitoring the presence of fire. The fire sensors are fixed on 3 sides of the robot so that it can detect fires at $2-3 \mathrm{~cm}$ range in the front of it and at its sides.

Once the robot detects fire the buzzer is made to turn $\mathrm{ON}$ and hence used to alert people of the existence of fire. Immediately the robot wheels stop spinning and the water pump gets activated, spraying water on all sides by turning the servo motor at 180 degree rotations .Hence keeping the fire under control.

\section{DESIGN AND ANALYSIS}

In this segment, the chosen equipment parts to be utilized for this task are investigated. Remember that the opposition imperatives were thought of as the configuration process was occurring.

\section{A. DC-Input}

There are $2 \mathrm{DC} 9 \mathrm{~V}$ batteries used here .One is used to power the arduino controller and the other is used to supply power to the sensors as well as the DC motors.

\section{B. Fire Sensor}

A flame sensor module consists of a flame sensor, resistor, capacitor, potentiometer, and comparator LM393 in an integrated circuit. It can sense infrared light with a wavelength ranging from $700 \mathrm{~nm}$ to $1000 \mathrm{~nm}$. The far-infrared flame probe converts the light detected in the form of infrared light into current. Sensitivity is optimized through the onboard variable resistor with a detection angle of 60 degrees. Working voltage is between $3.3 \mathrm{v}$ and $5.2 \mathrm{v} \mathrm{DC}$, with a digital output to show the presence of a signal. Sensing is conditioned by an LM393 comparator. The sensor outputs a digital low in case of Fire \& a digital high in all other cases.In our robot, we have utilized three of these fire sensors . One on the left, right and front side with the goal that the robot, when it moves identify fires on each of its three sides and subsequently is more compelling. The working reach is $2-3$ $\mathrm{cm}$

\section{Obstacle Sensor}

The operating voltage - 3-5.Detection range $-2-3 \mathrm{~cm}$ (adjustable using potentiometer) Outputs- HIGH in presence of obstacle. An IR sensor consists of an IR LED and an IR Photodiode; together they are called as Photo-Coupler or Opto-Coupler. As said before, the Infrared Obstacle Sensor has built in IR transmitter and IR receiver. Infrared Transmitter is an LED which emits infrared radiations. Hence, they are known as IR LED's. Even though an IR LED looks like a normal LED, the radiation emitted by it is not visible to the human eye. Infrared receivers are also called as infrared sensors as they detect the radiation from an IR transmitter. IR receivers come in the form of photodiodes and phototransistors. Infrared Photodiodes are different from normal photo diodes as they detect only infrared radiation. When the IR transmitter emits radiation, it reaches the object and some of the radiation reflects back to the IR receiver. Based on the intensity of the reception by the IR receiver, the output of the sensor is defined.

\section{Controller(ARDUINO UNO Board)}

Arduino is an open source computer hardware and software company, project, and user community. Arduino board use a variety of microprocessors and controllers. The boards are equipped with numerous sets of digital and analog input/output (I/O) pins that may be interfaced to various extension boards (shields) and other circuits. The boards feature serial communications interfaces, including Universal Serial Bus (USB) on some models, which are also used for loading programs from personal computers. The microcontrollers are programmed using an IDE based on the Processing language.

\section{DC-Motor}

DC Motors convert electrical energy into mechanical energy. The DC motor works on the principle of Lorentz force which states that when a wire carrying current is placed in a region having magnetic field, than the wire experiences a force. This Lorentz force provides a torque to the coil to rotate geared DC Motor has a gear assembly attached to the motor. The speed of motor is measured in terms of revolutions of the shaft per minute and is termed as RPM .The gear assembly helps in increasing the torque and reducing the speed. Using a combination of gears in a gear motor, its speed can be reduced to any desirable figure.

\section{E. L293D Motor Driver Module}

Fig 2: shows the Pin diagram details of L293D Motor Driver Module. It works by the principle of H-bridge. $\mathrm{H}$-bridge is a circuit which allows the voltage to be flown in either direction. Voltage need to change its direction for being able to rotate the motor in clockwise or anticlockwise direction, Hence H-bridge IC is ideal for driving a DC motor.

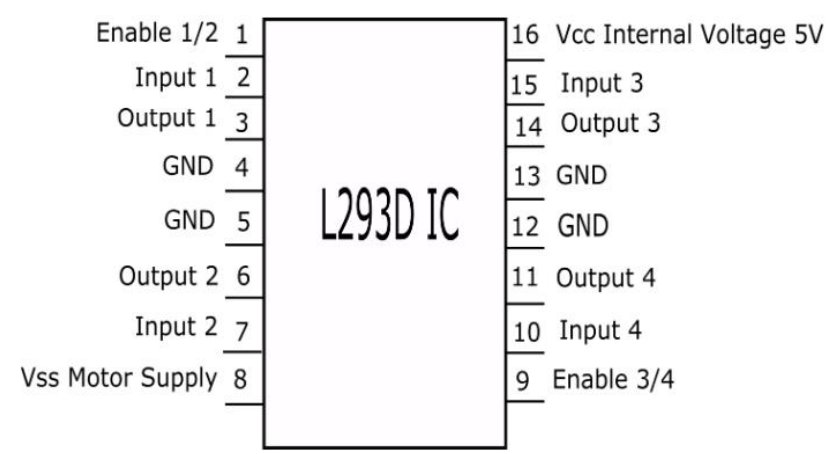

Fig. 2. Pin diagram of L293D Motor Driver Module

There are 4 input pins for L293D.Pins 2 and 7 on the left and pins 15 and 10 on the right are shown on the pin diagram. Left input pins will regulate the rotation of motor connected across left side and right input for motor on the right hand side. The motors are rotated on the basis of the inputs provided across the input pins as LOGIC 0 or LOGIC 1. 
TABLE 1:Working Mechanism Of L293d Motor Driver Module

\begin{tabular}{|c|l|c|}
\hline $\begin{array}{c}\text { Pin } \\
\text { Number }\end{array}$ & Pin Number & $\begin{array}{c}\text { Actions } \\
\text { performed }\end{array}$ \\
\hline Pin $2=1$ & Pin $7=0$ & Clockwise \\
\hline Pin $2=0$ & Pin $7=1$ & Anticlockwise \\
\hline Pin $2=0 / 1$ & Pin $7=0 / 1$ & No rotation \\
\hline
\end{tabular}

\section{F. Servo motor (SG90)}

It is a tiny and lightweight servo with high output power. It can rotate approximately 180 degrees (90 in each direction). The operating voltage of the servo is $5 \mathrm{~V}$ which is given with the help of a $9 \mathrm{~V}$ to $5 \mathrm{~V}$ reduction circuit using LM7805 IC along with a couple of capacitors to provide a stable $5 \mathrm{~V}$ from a standard $9 \mathrm{~V}$ battery.

\section{IMPLEMENTATION\& RESULTS}

The robot has been put together in 3 stages as follows

\section{A. Obstacle Avoidance}

An obstacle avoiding robot detects any obstructions in its path and avoids it by taking deroute from its current path.

The L293D motor driver is used to control the two motors. A motor driver is used because an Arduino cannot supply enough current to drive the motor.

The robot hardware connections are first made by connecting all the input output devices such as IR obstacle sensors, motors etc to the controller which is the ARDUINO board.

The controller is then programmed in such a way any one of the robots 2 obstacle sensors output the presence of an obstacle, depending upon the sensor detecting the obstacle the motor rotation direction is changed. If the left sensor detects an obstacle then the robot is made to move right by reversing the direction of rotation of the right motor. Similarly reverse is done if the right obstacle sensor detects an obstacle. If both the sensors detect the presence of an obstacle then the robot is made to move backwards by rotating both the wheels in reverse.

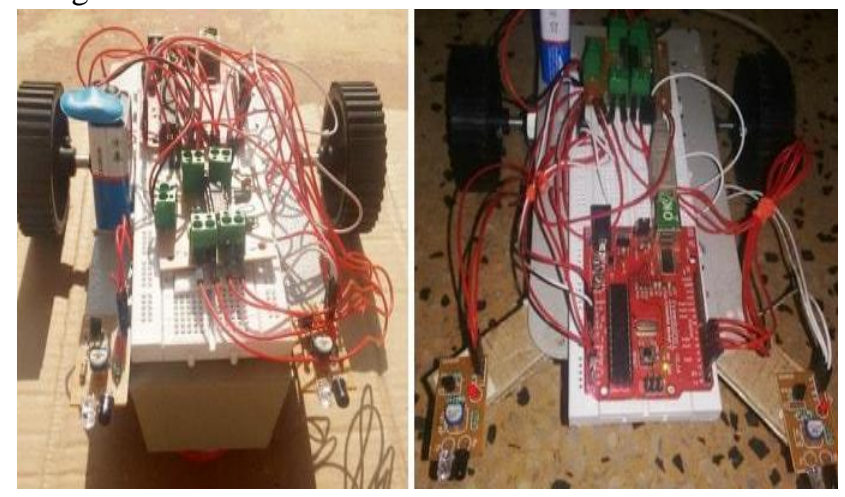

Fig. 3. Obstacle Avoidance Module

\section{B. Fire Detection}

It use flame sensor for detection and Arduino board for processing. 3 Flame sensors have been attached on the obstacle avoidance robot and a buzzer is also added in the circuit.

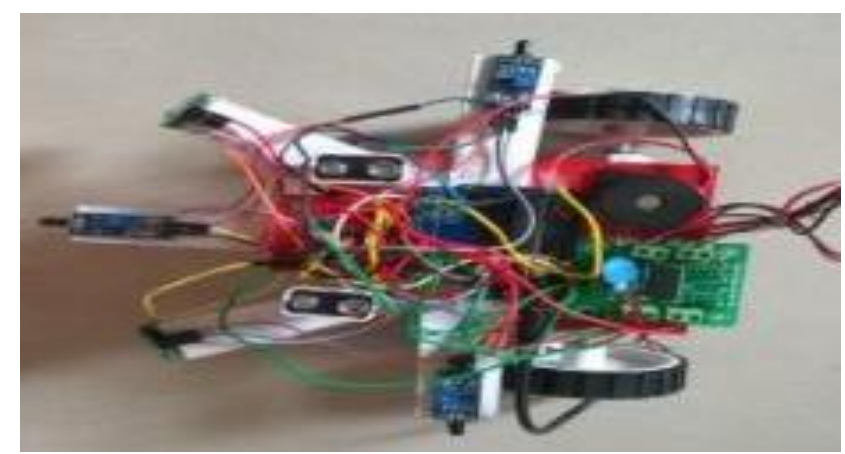

Fig. 4. Fire Detection Module

So the robot at this stage while moving with following obstacle avoidance will be noting for fire on its front, left and right side. Once fire is detected on any one of its sides, which is once any 3 of the fire sensors output the presence of a fire the obstacle avoidance is stopped.

This is done so that the obstacle avoiding censors don't detect the fire as an obstacle and hence move away from the flame. Now that the obstacle avoidance is not followed the robot is made to stand still and the buzzer at this stage is turned on for alerting people of the presence of fire.

\section{Keeping the fire in control}

In this stage of our Prototype we have used a $5 \mathrm{~V}$ water pump, a SG90 Servo motor and a container to carry water.

So when the fire is detected and the robot stops moving, the buzzer is sounded. The pump is turned on and the servo motor is programmed to move 90 degrees in the left and right direction with the water container along with the pump placed on top of it. So as the servo moves, the pump is turned on and the water present in the container is pumped out on the fire. Hence reducing the spread of fire and trying to extinguish it in case of a small fire.

If the fire is extinguished the alarm is turned off and if not the alarm is still kept ringing to indicate threat to people till the rescue teams arrive.

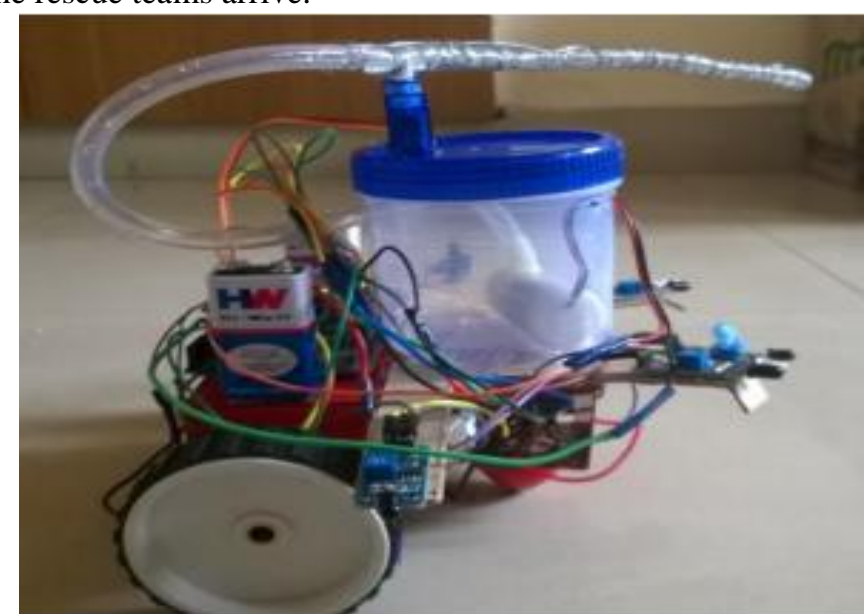

Fig.5.Photograph of the final automatic fire fighting robot 


\section{ALGORITHM}

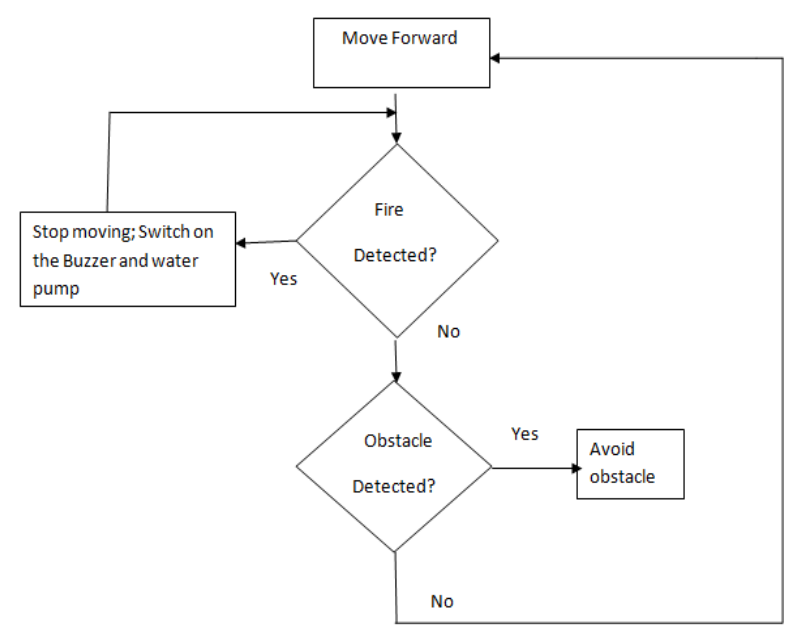

Fig. 6.The flowchart of the basic algorithm of the robot

\section{DIFFICULTIES FACED AND STEPS TAKEN}

When the robot used to detect fire, immediately the obstacle sensor also used to detect the fire as an obstacle and hence the robot used to move away from the fire, hence not making it possible for the water to fall on the fire. This has been completely rectified by changing the coding in such a way that if any of the fire sensors detect fire, then there obstacle avoidance code will not get executed. Hence making it possible to move closer. Also the sensitivity of fire has been increased and that of obstacle sensor decreased so that the fire is sensed before the obstacle.

The ARDUINO controller was getting over loaded because of the presence of too many input sensors and drives present, hence it heated the controller. This problem has completely been eliminated by giving an external supply for the sensors by stepping down 9V of battery using LM7805 IC.

In the prototype as the fire sensors used are based on IR principle, there is a problem where under extreme sunlight the IR fire sensors turn ON hence detecting and giving fake alarms. But as this project aims at making the best possible prototype model with using sensors and equipments available to us in the markets, the problems encountered in the hardware relating to sensors are out of scope. But at this point what we can come to a conclusion that the IR flame sensor we use has not been built to handle extreme sunlight as the detection range matches that of sunlight.

There was a problem in holding excess amounts of water $\&$ that the idea of extinguishing a huge fire with little water held on the robot looked impractical. But with the same reasoning used as before we eliminate this problem as being out of scope as we are aimed at only building a plausible prototype to the best extent possible. Hence the buzzer was included in the prototype model.

\section{FUTURE SCOPE}

This project has been motivated by the desire to design a system that can automatically sense fire and prevent it from spreading thereby putting-out the fire to the maximum extent possible on its own to protect life and property. The robot prototype that we have built can be extended by using advanced sensors, or rather invent sensors that can better detect fires and hence model a firefighting vehicle. GSM can be incorporated into the system and hence the location where the fire is present can be sent as a voice message to the fire department to call for help.

The robot can also be extended to make it like a hovercraft that can look into inaccessible spaces, detect fires and warn people of plausible dangers.

A live data video camera can also be attached to monitor what is going on in the areas affected by fire where the robot is moving and hence save many humans stuck in the fire breakout.

The robot can also be made to detect the kind of fire that it has detected and hence extinguish the fire based on the kind of fire for better efficiency.

If the robot needs to be made to move through and close to big fires then the robot can be upgraded and hence made with heat resistive materials like Nomex fiber or Nomex paper.

\section{APPLICATIONS}

- The robot can be used to detect fires in places humans cannot go into or in places where it might be dangerous for human intervention

- It can be used to alert residents, factories or electric companies regarding outbreaks of fire in their buildings so that they can safeguard valuable data, materials human life.

- This robot can be used to keep the fire in check and prevent its spreading until the firefighting agents come in.

- The robot can be used to guide people out or into places with fire breakout and hence leading them into safe paths and routs.

\section{CONCLUSION}

This paper has opened an interesting vision of the ideas which are used in this specific field. It plans to increase innovative advancement to accomplish a dependable and effective result from the different instruments. Experimental work had been carried out meticulously. The outcome demonstrates that higher productivity is for sure accomplished using the inserted framework. With a typical digitalized stage, these most recent instruments will empower expanded adaptability in charge, task, and development; take into consideration installed insight, basically cultivate the flexibility of the instruments; and in the long run advantage the clients with enhanced administrations, unwavering quality and expanded comfort. This paper show the significant highlight and elements of the different ideas that could be used in this field in detail through different frames. Since this base work cannot address everything inside the proposed system and vision, more innovative work endeavors are expected to completely actualize the proposed structure through a joint exertion of different substances. 


\section{REFERENCES}

1 Ahmed Hassanein, Mohanad Elhawary,Nour Jaber \& Mohammed Abd.(2015).Autonomous Firefighting Robot,American University of Kuwait.

2 Arvind Sanjeev, Nidhiya, V.,Raj, A free beginner's guide to making cool stuff using Arduino, E-Book, How to be a maker.

3 Playne, Matthew, Fire-Fighting Robot,Industrial Systems.

4 Sahil, S.,Shah,Vaibhav, K.,Shah,Prithvish Mamtora \& Mohit Hapani,. Aug,(2013). Fire Fighting Robot, IJETTCS journal .

5 Xu.,James,10Nov,(2013),(1989). Fire Fighting Robot. (2009). Industrial Systems Design and Integration.

610 Nov,(2013). Design \& Integration, (2006).

7 http://ieeexplore.ieee.org/document/7095886/

8 http://www.instructables.com/id/Arduino-Modules-Flam e-Sensor/

9 http://www.instructables.com/id/Autonomous-Fire-Fight er-Robot/

10 http://www.robotics.com/robomenu/index.html 